\title{
The Possibility of Spontaneous Gravitational Contraction of a Gas Cloud in Outer Space with Simultaneous Cooling of the Cloud
}

\author{
Alexey Belyaev \\ Independent Researcher, Russia
}

Copyright@2018 by authors, all rights reserved. Authors agree that this article remains permanently open access under the terms of the Creative Commons Attribution License 4.0 International License

\begin{abstract}
Gas clouds in outer space always lose energy due to electromagnetic radiation, i.e., are non-isolated thermodynamic systems. Under certain conditions, the energy loss of the gas cloud can lead to replacement of the process of dispersion by the process of spontaneous gravitational compression. Under these conditions of the initiation process of spontaneous compression, the gravitational energy released by the compression of the cloud will correspond to losses due to electromagnetic radiation it experiences. Furthermore, the cloud will shrink with a decrease in internal energy with constant enthalpy. As a result, the process of spontaneous gravitational compression of a gas cloud in outer space will be accompanied by a decrease in the temperature of the cloud. This mechanism of compression of a gas cloud with simultaneous cooling could, at a certain stage of the evolution of the universe, govern one of the possible processes of formation of a hidden mass of galaxies.
\end{abstract}

Keywords Gravitational Compression, Thermodynamic System, Hidden Mass

\section{Introduction}

We are all used to the fact that the compression of a gaseous medium leads to its heating, and the expansion of a gas is accompanied by a decrease in its temperature. We have gotten used to it because validity of this assumption has been established not only by theory and specially performed experiments, but also by everyday practice, for example, with the expansion of gas or steam in a turbine in the process of generating electricity, etc. The existence of stars in the universe is also associated with this: the cloud undergoing compression in outer space under the influence of internal forces of gravitation, inevitably warms up, becoming, under certain conditions, a star.
In the process of analyzing possible directions for the evolution of stars, Belyaev [1] emphasized that for a gas cloud located in outer space, i.e., for a cloud, whose dimensions are limited only by the forces of gravity, a stable equilibrium point cannot exist: for infinitesimal perturbations, the equilibrium gas cloud, whose internal pressure balances the internal gravitational forces, begins to either unlimitedly contract and self-heat or expand indefinitely and self-cool. In this case we are talking about an isolated system (the amount of energy lost with radiation is equal to the amount of external energy received in one way or another). However, it is obvious that for cosmic conditions, there can only be one possibility of such energy balance, which gives the right to consider a gas cloud as an isolated system, if we do not consider the cases of the emergence of controlled thermonuclear reactions, when the stars are ignited, that the initial stages of spontaneous compression of gas clouds with a temperature close to absolute zero.

The analysis of the compression of a gas cloud done by Belyaev in [1] demonstrated that the behavior of uninsulated systems differs from isolated systems, that the energy losses from the cloud contribute to the formation of degenerate structures. For model studies it is useful to consider the idealized conditions for the onset of compression, when the state of the cloud is not disturbed by turbulent processes. In existing models of the evolution of stars in the first stage of compression of a gas cloud, energy losses are determined only by electromagnetic radiation (Kelvin compression stage). Under Kelvin compression, a gradual self-heating of the diminishing cloud increases with simultaneous growth of losses due to electromagnetic radiation.

However, as it turns out, Kelvin compression is not the only possible variant of the beginning of compression of a non-isolated system, which is a calm gas cloud. Losses of energy with radiation can change, depending on the initial conditions, the very scheme of the compression of a gas 
cloud in outer space: compression under the influence of internal forces of gravitation can be accompanied not only self-heating, but also self-cooling (however, self-heating cannot be transformed into self-cooling by itself). This article is devoted to the study of this possibility for a closed non-isolated system.

\section{The Established Methodological Approaches in the Natural Sciences}

In the paper by Belyaev [1], in addition to the analysis of spontaneous compression of a gas cloud, another important topic, connected with the inattentive attitude to the detailed analysis of the phenomena described in modern natural sciences in physical terms (physics is to some extent present in all natural sciences) was also raised. The created models and theories are primarily justified mathematically, and only then, for the purpose of visualization of what has already been created, computer films are developed for demonstration. However, these films turn out as abstract as the basic theories underlying them.

Indeed, visualization is necessary. But it is necessary not at the stage of interpretation of the already created abstract model, but at the stage of developing the model itself. It is necessary for understanding the cause-effect relationships within the model itself. After all, the mechanical essence of the basic physical concepts used in all natural sciences, such as "energy", "work", "force", has not yet been cancelled (all basic physical categories were introduced into the theory at the stage of formation of corpuscular physics based on the law of inertia). And although it is not possible to create a mechanistic chain of interrelated events describing the studied phenomenon, it does not imply that the unsuitability of the mechanistic approach, but of the inadequacy of our fundamental knowledge. Nevertheless, even in these cases, it is necessary to continue studying the phenomenon in physical terms, rather than being content with only the mathematical description of the observed features of the described phenomenon.

The mechanistic approach is the basis of the philosophical view of "mechanicism"; the method of knowledge based on the fundamental principle of mechanical movement of elements of discrete matter in absolute space, taking place in absolute time according to the Newton laws under the influence of external forces and in compliance with the principle of inertia.

Galileo Galileo, Descartes and many others could be considered as the founders of mechanisism. Huygens, Hooke, Newton, Laplace, Hamilton (the latter indirectly, not because of the adherence to mechanism, but due to the scientific results achieved) and many others have significantly strengthened the positions of mechanicism. However, since the times of Hamilton, thanks to the scientific achievements of Lobachevsky, Riemann, Maxwell, Poincaré, Hilbert, and others, confidence in mechanicism has been undermined. Nevertheless, the method of cognizing this worldview, namely the mechanistic approach, still enjoyed confidence. However, the appearance of the Einstein's theories of relativity and quantum mechanics completely put an end to the mechanistic approach. Whether this is true is a topic for philosophers and the future development of science. In this case, it is being said that abolishing the cause-and-effect logic at the stage of development of the theory, based on physical principles is an obvious negative tendency of all natural sciences.

First a few words about physics. A mathematical description of the physical phenomenon is necessary. However, mathematics should be used to deepen the understanding of the essence of the physical model, not to create a mathematical model that is subsequently turned into a physical model. The mathematical justification of a physical model not only improves its understanding, but also allows for discovery of new manifestations of the physical phenomena. While the mathematical model has high chances to lead the theorist away from reality. At the same time, the construction of purely mathematical models, under which physical interpretations are selected, is also a useful. But it should be understood that such models do not have the right to be fundamental physical models, but only able to serve to better understand the harmony of true physical theories.

The serious problems emerging in the theory of physics associated with the prevailing disregard for the common sense based on the mechanistic interpretation will be discussed separately in future specialized works. The present work relates to the series of articles planned for publication and devoted to problems in astronomical sciences, identified on the basis of a general analysis in physical terms.

For example, today it is generally accepted that the birth of the universe is associated with the formation of a homogeneous cloud of matter expanding in space, which becomes, at the last stage of its existence, homogeneous hydrogen plasma. The prerequisite for such a conclusion was the non-stationary Friedman model, which attempted to describe the world of space-time mathematically. After the discovery, made by Hubble, of scattering matter in the universe, and the confirmation of Gamow's version of the existence of microwave background radiation, which was discovered by Penzias and Wilson, the Big Bang model based on the Friedman model was adopted as a model for the birth of the universe.

However, a general analysis in physical terms of the birth of the universe, carried out by Belyaev [2], showed that the Hubble law does not confirm the Friedmann mathematical model and the accepted Big Bang model, but, on the contrary, contradicts it. In addition, when Belyaev considered the properties of photon gas [3], also based on a general analysis in physical terms, it was shown that the discovered cosmic microwave background cannot be a 
relic.

In the late 1990s, in the process of refining the scale of distances in space, an accelerated dispersal of matter in the universe was discovered, which contradicted the generally accepted model of the Big Bang. However, the contradiction found did not encourage scientists to revise the accepted fundamental provisions in cosmology, but led to an unacceptable step for science: the premature introduction of a new category of "dark energy" into the theory. A new physical category allowed to save the Big Bang model, organically fitting into the model of cosmological inflation based on the Big Bang model. However, as a consequence a new fundamental problem arose: the need to define the physical essence of a physical category, the existence of which has never before been proven experimentally (except for the need to explain the accelerated dispersal of matter in the universe). Therefore, attempts to write a new physical category into a particular abstract mathematical model, rather than defining its essence by experimental means was a completely natural result of this approach. The unwillingness to set up experiments is explained by the impossibility of their implementation in laboratory conditions, because the energy, with its density distribution, of an unknown nature, evenly distributed in space, is only detectable on the scale of the universe. Therefore, the chances that someday dark energy from the abstract category will turn into a physical characteristic of vacuum, demonstrating at the mechanistic level the essence of the vacuum, are negligible. However, when studying the dynamics of compression of a dust cloud in outer space, performed again on the basis of a general analysis in physical terms by Belyaev [4], it was shown that the accelerated dispersal of matter in the universe can be explained within the framework of the classical theory of gravitation without introducing new categories.

The approaches adopted today for describing the evolution of the universe are clearly presented in the two-volume work of Gorbunov D.S. and Rubakov V.A. $[5,6]$. However, the results of the studies by Belyaev mentioned above [2-4] definitely point to the errors of the accepted cosmological model of the birth of the universe. At the same time, the evolutionary paths of the main astronomical objects, such as galaxies, stars and planets, depend on the cosmological model.

In order for a homogeneous cloud of matter to fragment into galaxies and stellar systems that are observed today, some instability conditions associated with a violation of the homogeneity of the matter density must arise. The characteristic dimensions of density perturbations that initiate the gravitational contraction of fragments in a homogeneous medium are called the Jeans length, which depends on the sound velocity in the medium, the density of matter, and the gravitational constant. It turned out that the mass of matter capable of separating into fragments at the expense of its own gravitational energy (the Jeans mass), initiates the formation of galaxies, and the stars are formed only as a consequence of the fragmentation of space into galaxies. Introduction of a new category of dark matter into the theory of cosmology did not change the adopted approach, however, it now became that the dark matter began to cluster in the galaxy and not matter. The results of Belyaev's works [1-4] allow us to initiate a conversation about the premature introduction of this new category into the theory. But this topic is above the scope of this paper and will have a more detailed coverage in another work.

Belief in a homogeneous cloud of matter at the first stages of evolution of the universe forced scientists to turn away from obvious physical facts that do not allow us to accept a quasar as the progenitor of galaxies. The reason for the appearance of the characteristic features of quasars and Seyfert galaxies is indicated in above mentioned work by Belyaev, who studies the gravitational compression of a dust cloud [4]. Moreover, the results of [4] imply that stars formed first and later they began to form galaxies, and galaxies formed into associations of galaxies.

But all these are common fundamental questions. If we return to Belyaev's work on the evolution of stars again[1], it clearly demonstrates that analysis in physical terms is always necessary, not only in the description of fundamental questions. This approach helps to better understand the resulting processes and even predict new nuances of manifestations of the studied phenomena. For example, the aforementioned paper it was demonstrated that the process of spontaneous compression of a gas cloud cannot stop without a qualitative change for the considered physical system, regardless of the initial mass of the gas cloud, its current temperature, and the gradual change in the chemical composition of the gas. Unfortunately, the phrases about the cooling of red and brown dwarfs, not to mention the lighter self-contracting gas clouds, can constantly be encountered in literature.

In-depth causal analysis in physical terms is so powerful that even when solving particular problems, it can still lead to fundamental questions. For example, in the paper by Belyaev [1] it is proposed to take a fresh look at the essence of degenerate structures and answer the question of the expediency of introducing a new astronomical term into the theory: "Absolute resistance to gravitational forces."

This paper, which considers the same object as in [1], namely, a quiescent self-contracting gas cloud in outer space, will continue the analysis begun in [1] and allow us to expand our knowledge of the spontaneous compression of gas clouds. It will show, based on general reasoning in physical terms, a new effect which has not been known before, because it does not self-excite under terrestrial conditions, and a goal needs to yet be set for conducting specialized experiments. However, under the conditions of outer space, this effect must inevitably arise and it is possible that at a certain evolutionary stage of the development of the universe it had a significant influence 
on the formation of a hidden mass.

\section{General Information on the Gravitational Compression of a Gas Cloud in Outer Space}

The forces of gravity are the weakest among all known fundamental forces. But on the scales of space they determine the fate of cosmic objects, since electric charges, in contrast to the "gravitational charge", have two signs. For this reason, the electric fields, stronger in comparison with the gravitational fields for each of the charges separately, are spontaneously mutually compensated and quenched at large distances from the clusters of matter or at large scales of matter accumulation.

All cosmic objects owe their existence to the gas, which was once released in the early stages of the evolution of the Universe, distributed in outer space. And gas, as is known, is a thermodynamic system. It is not the goal at this stage of the discussion, to describe the possible way of the appearance of a gas distributed in space and the shape of this distribution. The goal of this work is the description in physical terms of the behavior of gas as a thermodynamic system.

The description of thermodynamic systems is based on statistical laws that tend to redistribute the internal energy of the thermodynamic system so that an equilibrium state is established, unique for a closed and isolated system. For this reason, the category of "entropy" arises in thermodynamics, the main physical feature of which is spontaneous energy redistribution to an even distribution of the energy density in the space occupied by the thermodynamic system. However, this property of the thermodynamic system and the characteristic feature of the category "entropy" were not previously discussed in the literature and, therefore, are a separate topic of analysis. At this stage it is only important to emphasize that a closed and isolated thermodynamic systems always spontaneously tend to their unique equilibrium state. It is unique for each specific value of internal energy.

Strictly speaking, only a system with a large number of elements which in absolutely elastic and undamped collisions exchange momentums and energy and do not interact at a distance can be considered a thermodynamic system. In the presence of long-range forces that obey the principle of superposition (the total effect of the system on the element selected from it can be broken down into separate effects from each element surrounding it), the system can no longer be considered thermodynamic (such forces will violate the laws of statistics). Moreover, a system in equilibrium can be considered as a thermodynamic one. It's means that the system does not change its macro-characteristics for any long time. The laws of behavior of thermodynamic systems are determined through infinitesimal perturbations of their equilibrium states. Transitions between equilibrium states violate the laws of statistics, therefore, strickly, the system can be considered thermodynamic only in cases of slow transitions to new equilibrium states.

In [1], it was explained why a self-contracting gas cloud in outer space can be conditionally considered as a thermodynamic system, and it was mentioned that a self-contracting gas cloud, like a thermodynamic system, is described by the pressure of a gas cloud changing in accordance with the polytrophic law and seeking to balance the gravitational forces acting on each of the particles of the cloud. Depending on the compression conditions and gas properties, the polytrophic index can vary. At the polytrophic index $\gamma<4 / 3$, the gas cloud becomes unresisting to gravitational compression, since when the volume of the gas decreases, even by an infinitesimal amount, the growth of the gravitational forces exceeds the growth of the pressure resisting compression. The study of the pressure balance, which varies according to the polytrophic law, and the gravitational forces that depend on the distances between the elements of the system, makes it possible to understand the cause of the onset of the gravitational collapse, but does not describe the scheme of the occurring processes that, as it turns out, depends on the initial conditions.

In [1], in a schematic interpretation, it was shown that in a self-contracting gas cloud, in the absence of energy losses, the gravitational forces are primary, and it is the change in the forces of gravitation that leads to a change in the pressure in the cloud (the pressure changes only as a consequence of the change in the forces of gravity, and not spontaneously, causing a perturbation of the existing gravitational interactions). If, after the onset of spontaneous compression, energy losses occur, for example, when a hydrogen cloud compresses at a temperature close to absolute zero, the priority of forces does not change: the gravitational forces remain primary even in the changed situation when the system turns becomes a non-isolated system. However, it turns out that in the general case, not every uninsulated system retains this priority if the energy losses with electromagnetic radiation took place before the occurrence of spontaneous compression.

\section{Spontaneous Compression of a Gas Cloud in Outer Space, Accompanied by Self-cooling}

Let us consider a variant of an isolated gas cloud when the pressure in the cloud changes with the change in its volume according to the polytrophic law with the polytrophic exponent $\gamma=4 / 3$, in other words, when the internal energy of the gas cloud is equal to the field energy of the gravitational forces. This initial data implies that the polytrophic index remains largely unchanged with a high 
degree of accuracy when the volume of the cloud changes. Then the equilibrium point is indifferent to the current size of the cloud because changes in opposing forces of gravity and pressure occur synchronously equally. Let us look at how fair this assumption is on a figurative level.

It is known that the distribution of the parameters of the particles constituting the thermodynamic system follows simple laws of probability. Boltzmann established the general law of energy distribution between particles, but it was Maxwell who deduced that the distribution, taking into account the statistical weight, to determine the fraction of particles near the specific energy under consideration. Therefore, it is obvious that the most energetically charged particles of gas at the periphery of the cloud will leave it, as it happens when the liquid evaporates. Under the influence of the gravitational forces of the cloud, the escaped particles will start to decelerate and turn back to the cloud, but this will lead to collisions with the parts of the cloud flying towards them. This effect of "evaporation", inevitably accompanied by a decrease in temperature, leads to the usual dispersion of the cloud. The inevitability of dispersing a gas cloud, that does not lose energy in its environment (to outer space), in outer space leads to the fact that the cloud cannot have an equilibrium point, since it does not have a clear boundary, there is no shell enclosing the thermodynamic system, and forming a single system with it (the thermodynamic system must also be in equilibrium with the shell). Therefore, the free cloud, despite the zero balance of internal energy and energy of the internal gravitational field, will, firstly, constantly expand and, secondly, reduce its temperature. From the point of view of the law of conservation of energy, the decreasing gas temperature will be connected with the gas work of expanding against gravitation forces. But lowering the temperature reduces the resistance to the active revealing of internal gravitational compression forces, stimulating the system to change from expansion to compression. However, gravitation forces also decrease with the increase in distance. Therefore, the mental boundary of the equilibrium partition of a gas cloud with a vacuum will continuously expand, despite the cooling of the cloud, and the expansion process will continue until the temperature of the gas cloud drops to absolute zero (due to a decrease in the gas pressure, its condensation will not occur).

However, the non-isolation of the thermodynamic system can violated this picture. After all, a gas cloud in practice always loses its energy due to electromagnetic radiation. If the internal energy lowers due to losses, then the gravitational forces can take precedence over the expansion of the cooling cloud in the considered version of cloud expansion (for a closed and isolated system, with a turning point in the values of the polytrophic index), then the cloud will start to compress, trying to restore its temperature so as to return to conditions of unstable equilibrium. However, first, losses of internal energy take place only then the compression tries to reestablish the temperature. Under conditions of compression, when an unstable equilibrium state is disturbed by redirecting the energy from system with electromagnetic radiation, the gas inevitably turns into a liquid, which will then cool with crystallization. In other words, the compression of gas clouds under these initial conditions, when the energy of the gravitational field was equal to or slightly less than the internal energy, but the system is not isolated, occurs with final conversion of gas into a solid. However, gas clouds in space are composed mainly of hydrogen. And for the formation of solid hydrogen, very high pressures are assumed. Therefore, whether solid hydrogen can be formed from a hydrogen cloud compression with simultaneous heat removal is topic for a separate scientific study.

Since the released gravitational energy is caused by the attempts of the system to fully compensate for energy losses with electromagnetic radiation, which happens in practice with some delay in time, the work of gravitational forces will correspond to losses due to electromagnetic radiation, and the process of compression of a gas cloud with simultaneous decrease of its temperature will take place at constant enthalpy with a gradually decreasing internal energy. The work is done by forces of gravity because of the decrease in pressure inside the cloud caused by the decrease in temperature. However, the pressure depends not only on the temperature, but also on the volume. When losses with electromagnetic radiation occur, the thermodynamic system tends to maintain its temperature due to the compression process. However, continuing losses inhibit the desired increase in temperature and cause the need for additional compression in order to accelerate the growth of pressure by reducing the volume. As a result, the forces of gravity will tend to keep not the internal energy of the system, but its enthalpy, unchanged. The law of conservation of energy will be respected, the first law of thermodynamics as well, but why enthalpy and not the internal energy will be conserved is a very complex and important question. Therefore, it can only be considered within the framework of a separate work. At this stage, for the time being, one can only draw attention to the fact that the work in the steam power turbine is determined by the available heat drop, i.e,. is accomplished by changing the enthalpy of steam, and not by changing its internal energy.

Thus, on the basis of general arguments, without a mathematical description, which will need to be carried out subsequently for a comprehensive study of the indicated problem, an indisputable theoretical possibility of the existence of spontaneous gravitational compression of a gas cloud in outer space with its simultaneous cooling is shown.

In this case, the discussion is not about new laws or new theories. All thermodynamic processes, including the polytrophic processes, follow the laws of thermodynamics. The processes of heat loss with radiation also occur in 
accordance with the theory of radiant heat transfer. However, as it turns out, their joint manifestation can cause a physical phenomenon, which has not yet been discovered in observations. Gas is heated by compression, as is expected. However, the heating, which compensates for the heat losses that have already occurred, always occurs with a short delay, i.e., does not have time to restore the lowered temperature before the new losses of energy occur (the processes of compression and loss of energy with radiation are continuous, but in order to gain logical understanding of it, it is reasonable to break a continuous series into infinitesimal discrete portions). Due to the decrease in temperature, losses inevitably decrease with electromagnetic radiation. However, the rate of reduction in the volume of the system (the decrease in the geometric dimensions of the system with respect to the dimensions of the system at previous instants of time), which characterizes the process of compression with energy release, also decreases. Although compression slows down, the process of compression does not stop until the gas turns into liquid. Thus the possibility of the existence of a process of spontaneous compression of a gas cloud in outer space, which is an open thermodynamic system, with simultaneous spontaneous cooling is obvious.

However, a natural question of why such a process is not found in observations arises. Apparently, the following two things might be the reason.

First, for the emergence of the described situation, without sharp external excitations which form discrete gas clouds, not just massive clouds with a large potential of gravitational energy are needed to start the process of spontaneous compression, but also simultaneously sufficiently warm up so that losses from electromagnetic radiation are sufficient for the reversion of the process of expansion into the process of compression. But calm, i.e. undisturbed by turbulent disturbances, heated gas clouds are practically absent in interstellar space. Such favorable conditions could most likely develop only at the time of the formation of galaxies.

Secondly, if shock perturbing processes, like supernova explosions, create gas clouds in the space that begin to contract with self-cooling, then by astronomical observations it is impossible to establish at the initial stage of their compression the presence of the compression process, and in the course of their evolution such objects simply disappear from the observation field. However, theoretically, there is nothing that forbids the reproduction of such an experiment in terrestrial conditions.

\section{Conclusions}

The gas clouds in outer space, in which the intrinsic energy of gravity is slightly below or equal to the internal energy, always begin to expand regardless of their initial dimensions and mass, and the expansion of even an isolated system occurs with a decrease in temperature. Real clouds are not isolated systems and lose energy with electromagnetic radiation. Therefore, there are situations when, as a result of the energy losses from the cloud, the gravitational forces will begin to exceed the forces of pressure of the gas after a certain time interval, and the expansion process will be replaced by the compression process. The compression process, activated in this way, should not change the situation associated with a decrease in internal energy, and will also result from a decrease in temperature, but now of a shrinking cloud. The work done by gravitational forces will be equal to the energy loss with electromagnetic radiation, while the enthalpy of the system will remain unchanged, but the internal energy will be decreasing due to the modification of the statistical laws, which will cause a diversion from the energy system due to an initiating and supporting gravitational compression. Compression of clouds with simultaneous cooling can characterize one of the possible mechanisms of formation of a dark halo of galaxies and form a certain fraction of the hidden mass.

\section{REFERENCES}

[1] A. S. Belyaev, Trends of stellar evolution in modern physics. Universal Journal of Physics and Application, Horizon Research Publishing (HRPUB), vol. 11, No 6, 247-263, 2017. Online available from

http://www.hrpub.org/download/20171230/UJPA5-184107 84.pdf.

[2] A. S. Belyaev, A new model of the birth of the Universe. Universal Journal of Physics and Application, Horizon Research Publishing (HRPUB), vol. 11, No 5, 182-189, 2017. Online available from

http://www.hrpub.org/download/20170930/UJPA7-184102 01.pdf.

[3] A. S. Belyaev. Photon gas, Universal Journal of Physics and Application, Horizon Research Publishing (HRPUB), Vol.10, No.1, 11-15, 2016. Online available from http://www.hrpub.org/download/20160229/UJPA3-184060 08.pdf.

[4] A. S. Belyaev. Gravitational Contraction of a Dust Cloud in Space, Universal Journal of Physics and Application, Horizon Research Publishing (HRPUB), Vol.10, No.1, 22-30, 2016. Online available from

http://www.hrpub.org/download/20160331/UJPA5-184060 09.pdf.

[5] D. S. Gorbunov, V. A. Rubakov. Introduction to the Theory of the Early Universe: Hot Big Bang Theory, World Scientific Publishing Company, 2011.

[6] D. S. Gorbunov, V. A. Rubakov. Introduction to the Theory of the Early Universe: Cosmological Perturbations and Inflationary Theory, World Scientific, Singapore, 2011. 